\title{
Navigation in Multiobjective Optimization Methods
}

DOI:

10.1002/mcda.1599

\section{Document Version}

Submitted manuscript

Link to publication record in Manchester Research Explorer

\section{Citation for published version (APA):}

Allmendinger, R., Ehrgott, M., Gandibleux, X., Geiger, M., Klamroth, K., \& Luque, M. (2017). Navigation in Multiobjective Optimization Methods. Journal of Multi-Criteria Decision Analysis, 24(1-2).

https://doi.org/10.1002/mcda.1599

\section{Published in:}

Journal of Multi-Criteria Decision Analysis

\section{Citing this paper}

Please note that where the full-text provided on Manchester Research Explorer is the Author Accepted Manuscript or Proof version this may differ from the final Published version. If citing, it is advised that you check and use the publisher's definitive version.

\section{General rights}

Copyright and moral rights for the publications made accessible in the Research Explorer are retained by the authors and/or other copyright owners and it is a condition of accessing publications that users recognise and abide by the legal requirements associated with these rights.

\section{Takedown policy}

If you believe that this document breaches copyright please refer to the University of Manchester's Takedown Procedures [http://man.ac.uk/04Y6Bo] or contact uml.scholarlycommunications@manchester.ac.uk providing relevant details, so we can investigate your claim.

\section{OPEN ACCESS}




\section{Navigation in Multiobjective Optimization Methods}

\begin{tabular}{|r|l|}
\hline Journal: & Journal of Multi-Criteria Decision Analysis \\
\hline Manuscript ID & MCDA-15-0077.R1 \\
\hline Wiley - Manuscript type: & Research Article \\
\hline Complete List of Authors: & $\begin{array}{l}\text { Allmendinger, Richard; The University of Manchester, } \\
\text { Ehrgott, Matthias; Lancaster University } \\
\text { Gandibleux, Xavier; University of Nantes, UFR Science - LINA UMR CNRS } \\
\text { 6241 } \\
\text { Geiger, Martin; Helmut Schmidt University Hamburg } \\
\text { Klamroth, Kathrin; University of Wuppertal, Mathematics and Natural } \\
\text { Sciences } \\
\text { Luque, Mariano; University of Malaga, }\end{array}$ \\
\hline Keyword: & $\begin{array}{l}\text { Multiobjective optimization, multiple criteria decision making, preference } \\
\text { learning, navigation }\end{array}$ \\
\hline
\end{tabular}




\title{
Navigation in Multiobjective Optimization Methods
}

\author{
RICHARD ALLMENDINGER ${ }^{a}$, MATTHIAS EHRGOTT $^{b}$, XAVIER GANDIBLEUX $^{c}$, \\ MARTIN JOSEF GEIGER ${ }^{d}$, KATHRIN KLAMROTH $^{e}$, MARIANO LUQUE $^{f}$ \\ ${ }^{a}$ The University of Manchester, UK, ${ }^{b}$ Lancaster University, UK, ${ }^{c}$ Université de Nantes, France, \\ ${ }^{d}$ Helmut Schmidt University Hamburg, Germany, ${ }^{e}$ Bergische Universität Wuppertal, Germany, \\ ${ }^{f}$ University of Malaga, Spain
}

\begin{abstract}
Building on previous work of the authors, this paper formally defines and reviews the first approach, referred to as navigation, towards a common understanding of search and decision making strategies to identify the mostpreferred solution among the Pareto set for a multiobjective optimization problem. In navigation methods, the decision maker interactively learns about the problem, while the decision support system learns about the preferences of the decision maker. This work introduces a detailed view on navigation leading to the identification of integral components and features. A number of different existing navigation methods are reviewed and characterized. Finally, an overview of applications involving navigation is given, and promising future research direction are discussed.

KEY WORDS: Multiobjective optimization, multiple criteria decision making, preference learning, navigation
\end{abstract}

\section{Introduction}

Many optimization problems are made up of multiple, often conflicting, criteria. This leads to the loss of the formal but straightforward definition of optimality. Contrary to the existence of a single optimal solution, an entire set of Pareto optimal solutions (or trade-off solutions) might exist that 'optimizes' the considered criteria. Besides the complexity of solving multiobjective optimization problems (Miettinen, 1999), from a decision makers point of view, this raises the problem of making a selection of a single most-preferred solution.

Numerous different preference elicitation methods are available to facilitate the process of constructing representations of the decision makers' preferences (see e.g. Keeney and Raiffa (1976); Saaty (1977); Chen and $\mathrm{Pu}$ (2004)). Alternatively, there are techniques that allow an interactive search for a most- preferred solution without necessarily relying on the construction of an explicit notion of the actual preferences (see e.g. Korhonen and Wallenius (1988); Miettinen et al. (2010); Eskelinen et al. (2010); Hartikainen et al. (2015)). With the rise of human-machine interfaces, and the availability of powerful computer hardware, we foresee interactive search techniques to play an increasingly important role in the future. Consequently, some formal considerations of this research field are needed. Ultimately this should lead to a structuring of existing approaches and a stipulation of future research.

One way of approaching the above-sketched topic can be found in the introduction of the concept of navigation, which we will discuss based on the following informal definition in this paper:

Definition 1.1 (Navigation) Navigation is the interactive procedure of traversing through a set of points 
(the navigation set) in the objective space guided by a decision maker (DM). The ultimate goal of this procedure is to identify the single most-preferred Pareto optimal solution.

To the best of our knowledge, except our own preliminary research (Allmendinger et al., 2012) and the recent work of Hartikainen et al. (2015), which touches upon navigation methods for computationally expensive problems, the concept of navigation has not been considered in the literature yet and in particular not as formally and thoroughly as we do here.

Consequently, the contribution of this work is threefold: First, we describe the general framework of navigation methods, including the key aspects of navigation and various features according to which navigation methods can be characterized (Section 3). Second, we survey the current state-of-the-art in this research field by providing a summary of existing navigation methods as well as showcasing real-world applications of such methods (Section 4). Finally, we highlight several promising research directions of navigation (Section 5).

\section{Preliminaries}

This section provides the preliminaries and relevant background necessary to formally define navigation and put it in a proper context with existing research. For this we will provide the basic concepts of multiobjective optimization and an overview on how preference information can be accounted for in optimization. Finally, we will touch upon the importance of visualization in multiple criteria decision making (MCDM).

Definition 2.1 (Multiobjective optimization problem) The general formulation of a multiobjective optimization problem (MOP) is: "maximize" $\mathbf{f}(x)$ subject to $x \in X$, where $x$ is an n-dimensional candidate solution vector, $X$ is the search domain and $\mathbf{f}=\left(f_{1}, \ldots, f_{p}\right)$ is a vector objective function $\mathbf{f}: X \rightarrow \mathbb{R}^{p}$ mapping solutions to a $p$ dimensional real-valued objective space. The term 'maximize' is written in quotes in order to indicate that there are not unique maxima to such a problem in general, and a further definition is needed to define an ordering on candidate solutions (see below).

Definition 2.2 (Pareto dominance) Consider two solutions $x_{1} \in X$ and $x_{2} \in X$. We say that $x_{1}$ dominates $x_{2}$, also written as $x_{1} \succ x_{2}$, if and only if $\exists i$ such that $f_{i}\left(x_{1}\right)>f_{i}\left(x_{2}\right)$ and $\forall j, f_{j}\left(x_{1}\right) \geq f_{j}\left(x_{2}\right)$.

Definition 2.3 (Pareto optimal) A solution $x_{1} \in X$ is called Pareto optimal if there does not exist a solution $x_{2} \in X$ that dominates it. The objective function vector (or outcome) of $x_{1}$ is called a Pareto optimal outcome.

Definition 2.4 (Pareto front) The Pareto front (also known as Pareto set, Pareto frontier, or non-dominated set), denoted as $P F_{\text {true, }}$ is the set of all Pareto optimal outcomes or $\{\mathbf{f}(x) \mid x \in X, \nexists y \in X, y \succ x\}$.

From the above definitions it is apparent that to solve a MOP, the decision maker (DM) needs to compare several alternative non-dominated solutions and then select the single most-preferred one. Various preference-based approaches have been proposed in the literature to facilitate this procedure. Traditionally, these procedures have been classified as a priori, a posteriori, and interactive approaches referring to procedures where the DM provides preference information before, after and during the optimization, respectively (Branke et al., 2008).

A priori approaches allow the DM to specify the kind of desired solutions upfront but this means that the DM must have some form of understanding about the problem (e.g. range of feasible objective values, interdependcies of objectives) and her own preferences. Examples of preferences provided by the DM include reservation or aspiration levels (Haimes et al., 1971; Wierzbicki, 1980), or weights representing the relative importance of objectives (Triantaphyllou, 2000). In contrast, a posteriori approaches first approximate or compute the Pareto front (or a part of it) using e.g. evolutionary multiobjective optimization techniques (Deb, 2001), and then ask the DM to select the most-preferred solution.

Interactive approaches combine optimization with 
preference handling speeding up the convergence to the most preferred solution (Branke et al., 2008). Typically, such approaches assume a mathematical model of the DM's preferences, which is build up during the search procedure as preference information from the DM becomes available. A commonly used approach to build up the preference model (value functions) is to prompt the DM to perform pairwise comparisons of solutions (Greenwood et al., 1997; Branke et al., 2010). Although effective in converging to the single most-preferred solution, such methods can be computationally expensive. Recent research (e.g. (Branke et al., 2016, 2015)) investigated alternative methods to minimize this computational burden.

It is apparent that both a posteriori and interactive methods allow the DM to somehow traverse (or navigate) through a set of points to identify a single mostpreferred solution. An integral aspect of this process, and when solving a MOP in general, is that a DM needs to compare several candidate solutions before selecting the most-preferred one (Ruiz et al., 2015b). This task becomes more challenging as the number of objectives in the MOP increases ${ }^{1}$, urging the need for decision support tools.

Graphical visualization tools (Lotov et al., 2005; Lotov and Miettinen, 2008) are one example of such tools, and also an integral part of the navigation framework. A recent survey on this topic that provides an overview of various visualization techniques, such as techniques using bars, value paths, circles, polygons, icons, and trees, has been presented in Miettinen (2014). Note that while these techniques may find application when dealing with small sets of candidate solutions (around ten or less), large solution sets may first undergo a pre-processing (or information reduction) step before using these techniques (or require different tools altogether). Examples of commonly used pre-processing steps include clustering (Malakooti and Raman, 2000) and multivariate analysis techniques (Gnanadesikan, 2011).

\footnotetext{
${ }^{1}$ When deciding how many solutions to show to a DM (e.g. for ranking or selection) or how many objectives to optimize simultaneously in a MOP, it may be worth keeping in mind the magical number seven plus or minus two (Miller, 1956). This number has been established via a series of psychological tests investigating the limits of human beings in receiving and processing of information.
}

It will be apparent in the following sections that visualization is a core element of a navigation method, and that developers of such methods are beginning to work more closely with visualization and analytics experts to create more intuitive and efficient graphical user interfaces (GUIs) to support the decision-making process.

\section{Navigation Framework}

Following the rather general definition in the introductory section, some more precise definitions and elaborations are needed in order to fully understand the concept of navigation. Figure 1 sets the foundation of the framework by illustrating the concept of navigation, how it can be embedded in an IT-landscape, and its relation to preference learning. A general framework consists of three levels: a solving component, a learning component, and the navigation process itself, all of which are embedded in a "computing cloud". The solving component captures the models, algorithms, and data needed to create the navigation set. The learning component is responsible for the preference modelling and learning with the aim to establish a rich set of preference information. The navigation process itself communicates between the solving and learning component via a GUI. This general framework captures the structure of several existing navigation methods (as will be described in Section 4.1) and is also suitable in the context of computationally expensive optimization problems (Hartikainen et al., 2015).

The following two sections will elaborate about this framework and key features of navigation in more detail.

\subsection{Key Aspects of Navigation}

Here we discuss several aspects that need to be addressed to define a navigation procedure.

\section{What is the set to be navigated?}

Three types of sets to be navigated can be distinguished: (i) the entire Pareto front, (ii) a true subset of the Pareto front, or (iii) any other set of points. Consequently, navigation can be combined with a posteriori approaches in which the 


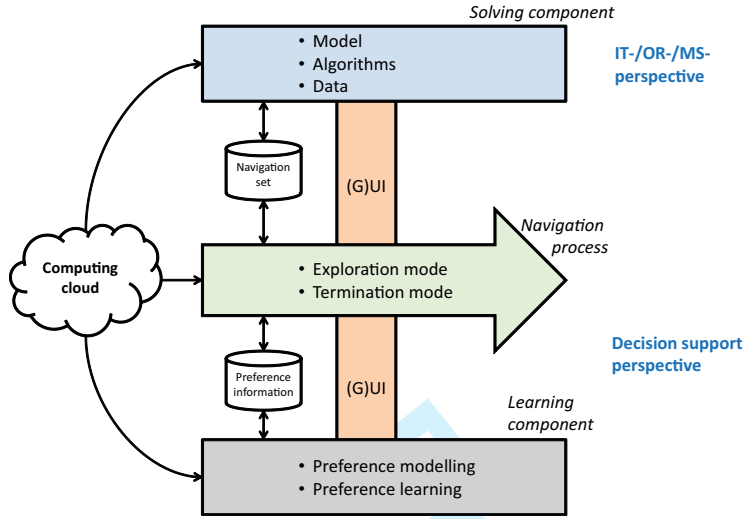

Figure 1: A framework of navigation and its embedding into a commercial IT-landscape.

Pareto front is first identified/approximated before the navigation phase. A further interesting case is found in many (classical) interactive approaches, which consider a single outcome and progress from there.

\section{How to navigate?}

Navigation can be thought of as the following iterative procedure: Upon a DM's action, e.g. specification of a desired navigation direction, the system reacts such that new points are selected/computed in real time and presented to the DM. This loop of dialog- and move-phases is repeated until the DM has identified the most preferred solution in the navigation set. Figure 2 depicts this interactive procedure in form of a flowchart.

Note that while navigation takes place in the objective space, the DM can also take information from the decision space into consideration to affect guidance through the navigation set.

\section{Guidance provided by the navigation}

During a navigation procedure, the DM can request guidance from the system. Two modes can be distinguished: exploration mode and termination mode.

During the exploration mode, the control is fully in the hand of the DM. In this mode, the DM learns about the problem. Guidance/support provided by the method can facilitate this process using, for example: (i) cycle detection, (ii) information about the possible alternatives, (iii) direction derived from the navigation history, (iv) statistics of the navigation history, (v) intensification/diversification characteristics of navigation steps.

The termination mode is entered when the DM requests strong support from the system in order to be convinced that the most-preferred solution has been found. This information could be provided by the use of value functions, which are e.g. extracted from the statements made by the decision maker. In this mode, the system learns from the DM.

\subsection{Features of Navigation}

Based on the integral properties of navigation, several features arise that need to be considered prior or during the interactive search:

1. Navigation in the Pareto vs. non-Pareto set? On the one hand, when navigating in the Pareto set only, any navigation direction implies the worsening of at least a single objective. On the other hand, navigating in the non-Pareto set may allow for a simultaneous improvement without 'sacrificing' the current values. This has some implications for possible navigation directions resulting from the actions taken by the DM. In any case, and ultimately, the final outcome of navigation should be a point of the current navigation set for which no other point known dominating it is known.

2. Does the starting point of the navigation procedure affect the final solution reached?

A key question is whether the same ultimate point is reached when starting from different points. We believe this to be the case if certain assumptions are made with respect to the value function of the DM, the consistency of the navigation and the preference/direction statements. 


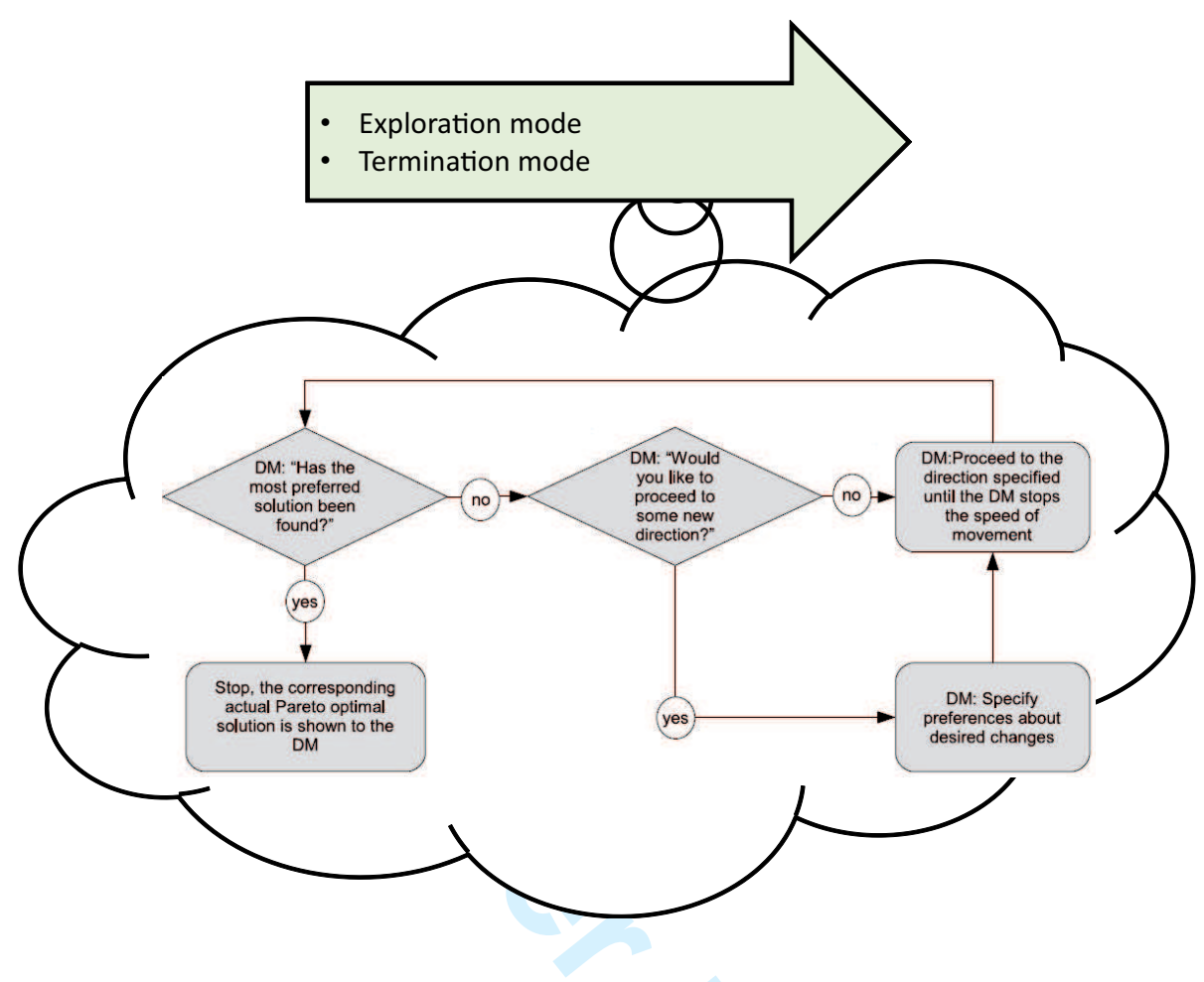

Figure 2: Interactive application of navigation with a DM including move- and dialog-phases. The flowchart has been reproduced from (Eskelinen et al., 2010).

3. How to account for behavioral aspects (e.g. inconsistent behavior)?

Especially in the exploration phase, a certain amount of 'inconsistent' behavior is to be expected. This stems from the fact that the DM explores the navigation set in order to learn about the problem. As a consequence, any method implementing navigation should account for this issue. Following the implications from prospect theory (Kahneman and Tversky, 1979), a DM may not judge symmetrically with respect to gains and losses of previously obtained outcomes. Navigation methods can take this into account by selecting a dominated starting point. This is the approach taken, for example, by the Nautilus approach (Miettinen et al., 2010), which will be described in more detail in Section 4.1.

\section{Is the problem of discrete or continuous nature?}

The precise properties of the problem are important. Whether the considered problem is discrete or continuous influences the type of navigation that can be used. In both cases, discrete representations satisfying different aspects (e.g. hypervolume, uniformity, coverage, and approximation error) can be used as the basis for navigation. Similar considerations may need to be made depending on whether the problem is linear vs nonlinear and convex vs nonconvex.

\subsection{Desirable Properties of Navigation}

Depending on the problem type, the available information about the problem and the DM's preferences, and the envisaged navigation concept, a navigation method can be evaluated based on different quality measures. In the context of nonconvex and computationally expensive optimization problems, an exhaus- 
Table 1: Characterization of existing navigation methods.

\begin{tabular}{|c|c|c|c|c|c|}
\hline $\begin{array}{l}\text { Navigation } \\
\text { method }\end{array}$ & Navigation set & $\begin{array}{l}\text { Type of so- } \\
\text { lution pro- } \\
\text { cedure }\end{array}$ & $\begin{array}{l}\text { Nature } \\
\text { problem }\end{array}$ & $\begin{array}{l}\text { Graphical user interface } \\
(G U I)\end{array}$ & $\begin{array}{l}\text { Navigation actions avail- } \\
\text { able to DM }\end{array}$ \\
\hline $\begin{array}{l}\text { Pareto race (Ko- } \\
\text { rhonen and Wal- } \\
\text { lenius, 1988) }\end{array}$ & Pareto front & Interactive & $\begin{array}{l}\text { Linear and } \\
\text { quadratic- } \\
\text { linear (Ko- } \\
\text { rhonen and } \\
\text { Yu, 1997) }\end{array}$ & $\begin{array}{l}\text { Current objective function } \\
\text { values in numerical form } \\
\text { and as barcharts }\end{array}$ & $\begin{array}{l}\text { Update of navigation direc- } \\
\text { tion (reference point) and } \\
\text { speed (step size) }\end{array}$ \\
\hline $\begin{array}{lr}\text { Database } & \text { ap- } \\
\text { proach } & \text { (Thieke } \\
\text { et al., 2007; } \\
\text { Ehrgott and } \\
\text { Winz, 2007) }\end{array}$ & $\begin{array}{l}\text { Finite subset } \\
\text { of Pareto front }\end{array}$ & $\begin{array}{l}\text { A posteri- } \\
\text { ori }\end{array}$ & Convex & $\begin{array}{l}\text { Application-specific but typ- } \\
\text { ically min and max objective } \\
\text { values in database, current } \\
\text { objective function values }\end{array}$ & Navigation direction \\
\hline $\begin{array}{l}\text { NAUTILUS (Mi- } \\
\text { ettinen et al., } \\
2010)\end{array}$ & $\begin{array}{l}\text { Outcomes of } \\
\text { feasible and } \\
\text { infeasible non- } \\
\text { Pareto optimal } \\
\text { solutions (but } \\
\text { final solu- } \\
\text { tion is Pareto } \\
\text { optimal) }\end{array}$ & Interactive & $\begin{array}{l}\text { Linear and } \\
\text { non-linear }\end{array}$ & $\begin{array}{l}\text { Estimates of ideal and nadir } \\
\text { vectors, current objective } \\
\text { function values in numerical } \\
\text { form and as barchart, current } \\
\text { lower bound objective vec- } \\
\text { tor }\end{array}$ & $\begin{array}{l}\text { Number of solutions visited } \\
\text { during navigation, prefer- } \\
\text { ence information (rank im- } \\
\text { portance of each current ob- } \\
\text { jective value OR specify } \\
\text { percentages reflecting 'like- } \\
\text { ness' of improving the cur- } \\
\text { rent objective values) }\end{array}$ \\
\hline $\begin{array}{l}\text { Pareto naviga- } \\
\text { tor (Eskelinen } \\
\text { et al., 2010) }\end{array}$ & $\begin{array}{l}\text { Polyhedral } \\
\text { approximation } \\
\text { of the Pareto } \\
\text { front }\end{array}$ & $\begin{array}{l}\text { A posteri- } \\
\text { ori }\end{array}$ & $\begin{array}{l}\text { Non-linear } \\
\text { convex } \\
\text { and mildly } \\
\text { nonconvex }\end{array}$ & $\begin{array}{l}\text { Estimates of ideal and nadir } \\
\text { vectors (or the DM provides } \\
\text { these vectors), evolution of } \\
\text { objective function values in } \\
\text { form of a value paths and } \\
\text { barcharts }\end{array}$ & $\begin{array}{l}\text { Update of navigation direc- } \\
\text { tion (reference point) and } \\
\text { speed (step size) }\end{array}$ \\
\hline $\begin{array}{l}\text { Nonconvex } \\
\text { Pareto naviga- } \\
\text { tor (Hartikainen } \\
\text { et al., 2015) }\end{array}$ & $\begin{array}{l}\text { PAINT } \\
\text { method-based } \\
\text { approximation } \\
\text { of the Pareto } \\
\text { front }\end{array}$ & $\begin{array}{l}\text { A posteri- } \\
\text { ori }\end{array}$ & Nonconvex & $\begin{array}{l}\text { Estimates of ideal and nadir } \\
\text { vectors (or the DM provides } \\
\text { these vectors), evolution of } \\
\text { objective function values in } \\
\text { form of a value paths }\end{array}$ & $\begin{array}{l}\text { Update of navigation direc- } \\
\text { tion (reference point and ob- } \\
\text { jective bounds) and speed } \\
\text { (step size) }\end{array}$ \\
\hline $\begin{array}{l}\text { E- } \\
\text { NAUTILUS (Ruiz } \\
\text { et al., 2015a) }\end{array}$ & Pareto front & $\begin{array}{l}\text { A posteri- } \\
\text { ori }\end{array}$ & Nonconvex & $\begin{array}{l}\text { Estimates of ideal and nadir } \\
\text { vectors (or the DM provides } \\
\text { these vectors), evolution of } \\
\text { objective function values in } \\
\text { form of a value paths and } \\
\text { barcharts }\end{array}$ & $\begin{array}{l}\text { Number of intermediate } \\
\text { points and total number } \\
\text { of steps in the navigation } \\
\text { process }\end{array}$ \\
\hline
\end{tabular}

tive list of desirable properties is suggested in Hartikainen et al. (2015). This includes technical properties like completeness of the navigation (i.e. every non-dominated point should be at least theoretically reachable), computational efficiency and approximation quality, as well as properties related to the user experience like navigation control, the cognitive load on the DM and the availability of additional information.

Copyright (C)2015 John Wiley \& Sons, Ltd.

\section{Previous Research Related to Navigation}

Although the concept of navigation has not been defined and characterized in the literature as concretely as we do here, several approaches incorporating this concept have been proposed in the last three decades. This section gives an overview of these approaches, and also outlines several application domains where 
navigation methods have been applied or where we envisage that such methods would be beneficial.

\subsection{Existing Navigation Methods}

This section describes several seminal navigation methods and links them to the key aspects and features introduced in Section 3.2. Table 1 provides a summary of the methods. The methods are described in the order of their appearance highlighting the surge in complexity and sophistication of the methods and the IT-landscape they have been embedded in.

Pareto race: Korhonen and Wallenius (1988) developed arguably the first dynamic and visual interactive search method to solve multiple objective linear programming problems. This method, which the authors called Pareto race, allows the DM to search freely through the Pareto front. Interaction between the optimizer and the DM is ensured through a simple yet intuitive user interface (see Figure 3). The interface displays the objective function values in numeric form and as bar graphs whose lengths adapts dynamically as the DM searches through the Pareto front. Keyboard controls enable the DM to dictate speed and direction of motion of the search. Pareto race exploits the DM's aspirations through an achievement (scalarizing) function (Wierzbicki, 1980). Starting from a feasible solution $x^{*}$, this function is used to project a reference direction on the set of (weakly) Pareto optimal solutions by solving a parametric linear programming problem. An update of the DM in the search configuration changes this problem accordingly, thus enabling a dynamic and real time search. Pareto race is inspired by the visual reference direction approach to multiobjective linear programming developed by Korhonen and Laakso (1986b,a) but is more dynamic due to the implicit definition of the reference direction. Pareto race has been extended in a number of ways, such as to multiple objective quadratic-linear programming problems (Korhonen and Yu, 1997) and more recently to nonlinear convex problems (Eskelinen et al., 2010); this approach will be explained in more detail later in this section.

Database approach: What we call the database ap-

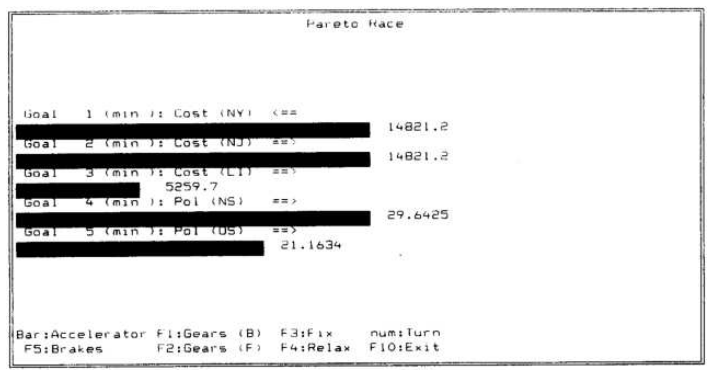

Figure 3: Graphical user interface of Pareto race. Screenshot taken from (Korhonen and Wallenius, 1988).

proach to navigation is closely related to the concept of finite representation of the Pareto front of a multiobjective optimization problem. If $P F_{\text {true }}$ denotes the Pareto front of a multiobjective optimization problem (see Definition 2.4), then a finite subset $R \subset P F_{\text {true }}$ is called a finite representation of $P F_{\text {true }}$. The name indicates that, for the purposes of decision-making, $R$ can be considered instead of $P F_{\text {true. }}$. Under this hypothesis, the decision making process is reduced from the selection of a most preferred outcome from an infinite set to selection from a finite set. Moreover, this selection from a finite set is supported by a large number of methods in multi-criteria decision aid, see e.g. Greco et al. (2016). This reduction to selection from a finite set can, however, only be justified if the representative set $R$ satisfies certain quality criteria. Subsequent to the work of Sayın (2000), the most commonly considered criteria are cardinality (the size of $R$ should not be too large), coverage (every area of the Pareto front should be represented) and uniformity (the representative points should be uniformly distributed across the Pareto front). Since a survey of methods for the computation of representative sets is beyond the scope of this paper, we refer to Faulkenberg and Wiecek $(2010,2012)$ and references therein.

For the purpose of this work, it is sufficient to note that, since $R$ acts as a substitute of the entire Pareto front, we can consider the underlying solutions, the outcomes of which form the set $R$, as a database of 


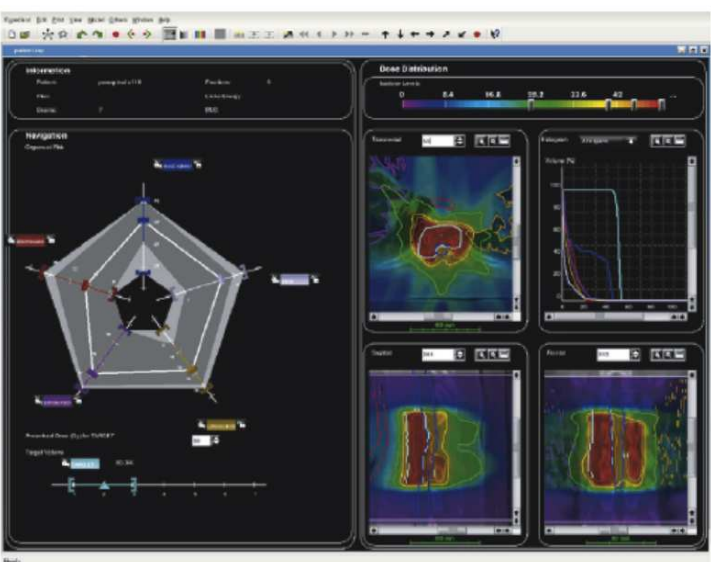

Figure 4: Graphical user interface of the Database approach as used in a radiotherapy treatment design problem. Screenshot taken from (Thieke et al., 2007).

solutions from which the DM needs to select a most preferred solution. The search for a most preferred solution can then be restricted to a search among the database. Since the elements of the database have been computed offline without the involvement of a DM, no further optimization is necessary, and the selection process can be handled online. This allows the use of graphical user interfaces, visualization tools and other techniques as appropriate for an application. Figure 4 shows an example of a graphical user interface as used in a radiotherapy treatment design problem (Thieke et al., 2007), which will be explained in more detail in the next section.

NAUTILUS: NAUTILUS (Miettinen et al., 2010) is an interactive method based on an unusual navigation set consisting of points that can be feasible or infeasible and where all points are dominated by at least one non-dominated point except the last point. This last point lies on the Pareto front and, given the DM acts rationally, is the most preferred point. Plenty of the interactive methods for multiobjective optimization are based on the sequential determination of nondominated points by introducing new preferential information at each iteration. This means that the DM must always allow the impairment of at least one ob-

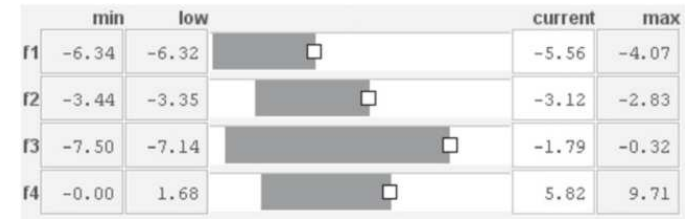

Figure 5: Graphical user interface of NAUTILUS. Screenshot taken from (Miettinen et al., 2010).

jective function to produce the next iteration. The main purpose of NAUTILUS is to eliminate the 'sacrifice' of at least one objective function at each iteration, due to the psychological assumption that people do not react symmetrically to gains and losses. Another important purpose is to avoid the anchoring effect mainly due to the starting point.

In this method, each solution dominates the previous one, whereupon the navigation is always carried out improving all objective functions in a given direction. This direction is obtained through the consideration of preferential weights that reflect the DM's preferences and where, by minimizing an achievement scalarizing function, the search is oriented towards the part of the Pareto front that the DM prefers. In this navigation process, the user interface shows the DM the range of the attainable values for each objective function (see Figure 5) at each iteration (upper and lower bounds). These ranges are contracted at each iteration of the navigation procedure and thus guide the search towards the desired part of the Pareto front.

Pareto navigator: Pareto navigator (Eskelinen et al., 2010) extends the ideas of Pareto race (Korhonen and Wallenius, 1988) to nonlinear convex and mildly nonconvex problems with multiple objectives, and works as follows:

1. In a pre-processing (initialization) phase, a convex polyhedral approximation of the non-dominated set is computed using an appropriate approximation method. In this way, the Pareto race concept can be transferred to nonlinear problems, and expensive objective function evaluations can be avoided during the interactive navigation phase.

2. After specifying an initial solution/point (e.g., from 


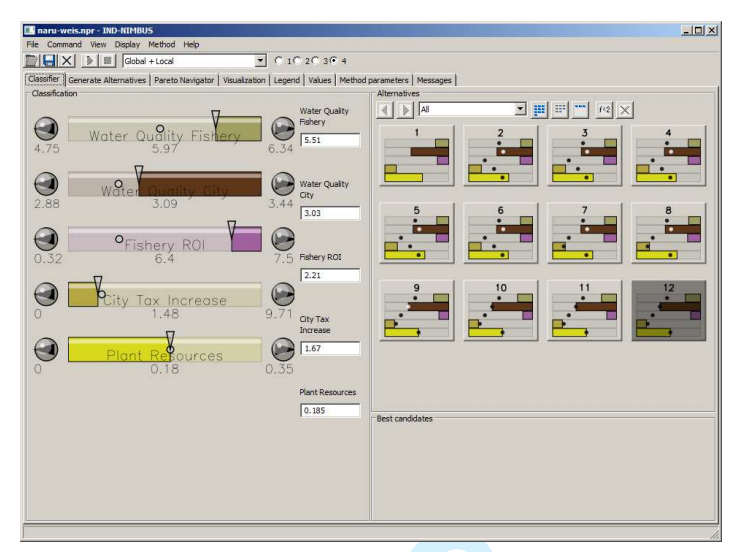

Figure 6: Graphical user interface of Pareto navigator. Screenshot taken from (Eskelinen et al., 2010).

the previously computed approximation), the DM can explore the non-dominated set and collect trade-off information by navigating in the polyhedral approximation. In each iteration of this navigation phase, the DM specifies a search direction, for example, by a classification approach or by directly specifying a reference point. The movement towards this direction is realized using parametric linear programming on the polyhedral approximation, and is visualized using, for example, value paths with appropriate step lengths (see Figure 6).

3. At any time during the navigation, the DM can change the speed of the movement, the direction, or request the computation of the closest non-dominated point. This point can then be included in the approximation and the search can be continued, or the DM may choose to terminate the search at this point.

When the DM has completed the learning phase with the Pareto navigator, he or she may wish to continue with some other interactive method to complete the termination phase, or simply stop with the final solution found.

E-NAUTILUS: Enhanced NAUTILUS (ENAUTILUS) (Ruiz et al., 2015a) is based on the NAUTILUS method (Miettinen et al., 2010) and shares the same motivation of avoiding trade-off and anchoring bias. It can be seen as the a posteriori version of NAUTILUS and applies a three-stage solution process: In the first stage, the pre-processing step, an a posteriori type method or evolutionary multiobjective optimization algorithm is used to pre-compute a well-spread Pareto set; for a recent review on evolutionary-based interactive and $a$ posteriori decision making methods, please refer to Purshouse et al. (2014). This is followed by the interactive decision making stage, where, based on the pre-computed solution set, the DM interacts with the solution process until a single-most preferred solution is found. In the final stage, the post-processing stage, it is ensured that the Pareto optimality of the final solution is guaranteed. Prior to the optimization, the DM specifies the number of points (intermediate points) (s)he wants to be shown at each step of the navigation process, and the total number of steps in the navigation procedure. At each step of the navigation process, it is ensured that the intermediate points are closer to the set of Pareto optimal solutions. Thanks to the pre-processing stage, E-NAUTILUS does not need to solve a new optimization problem at each step of the navigation process, preventing the DM from having to wait for the presentation of a new solution set. This makes E-NAUTILUS more suitable for problems with expensive evaluations.

Nonconvex Pareto navigator: Nonconvex Pareto navigator (Hartikainen et al., 2015) extends the ideas of the Pareto navigator (Eskelinen et al., 2010) to nonconvex problems. The approximation of the Pareto front (generated in the initialization phase) is based on the PAINT method (Hartikainen et al., 2012) applied to a set of precomputed non-dominated points. The resulting piecewise linear approximation contains all given non-dominated points and is consistent in the sense that it is inherently non-dominated. To facilitate navigation between different and possibly unconnected parts of this approximation, it is extended by enlarged dominance cones that contain the positive orthant.

The advantage of adding enlarged dominance cones to the approximation is two-fold: On the one hand, it is shown that (under appropriate assumptions on the choice of the cones) the approximation provides a complete representation of the non-dominated set. 


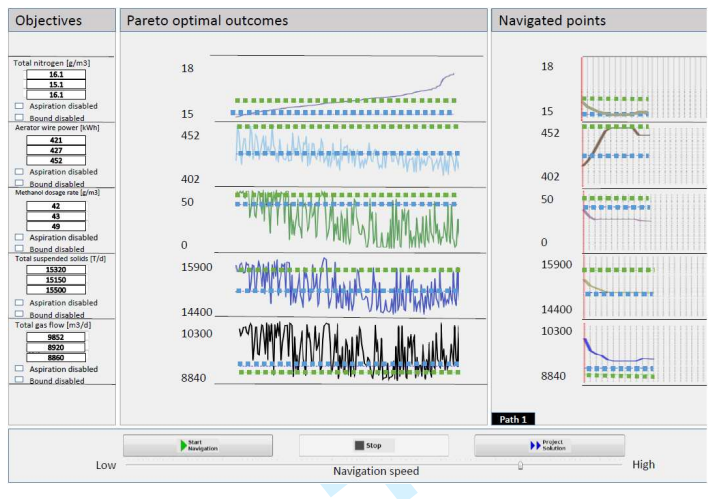

Figure 7: Graphical user interface of Nonconvex Pareto navigator. Screenshot taken from (Hartikainen et al., 2015).

On the other hand, these cones can be used to integrate information on favorable trade-offs into the model. The resulting piecewise linear approximation is connected and can be represented by a mixed integer linear programming problem. The navigation phase combines ideas from the Pareto navigator (Eskelinen et al., 2010) with classification-based preference models inspired by the NIMBUS method (Miettinen and Mäkelä, 1995). Figure 7 shows the modern and informative graphical user interface of this approach.

\subsection{Applications}

Having outlined several seminal navigation methods in the previous section, in this section we discuss various problems and application domains to which navigation has been applied or where we envisage scope for its application in future.

Radiotherapy treatment design: The goal of external radiation therapy of cancer is to eradicate tumor cells by delivering ionizing radiation from an external source to the tumor without compromising surrounding normal tissue and organs at risk (OAR). Due to the physics of radiation delivery, there exists a tradeoff between the conflicting goals of tumor control and the avoidance of side effects in surrounding normal tissue. The most common technique used today is inten- sity modulated radiotherapy (IMRT), which allows the modulation of radiation intensities through the use of a mechanical device called multi-leaf collimator (MLC).

For about the last two decades, optimization methods have been applied to assist oncologists in the design of IMRT treatments. Optimization models for the selection of beam angles, fluence maps, and delivery sequences are well known in the literature, see e.g. Ehrgott et al. (2009) and references therein. The problem of interest here is the so-called fluence map optimization problem, which consists in the determination of the modulated intensity patterns for a number of pre-selected beam angles that, once delivered by the (MLC) achieve the goals of maximizing tumor control and minimizing normal tissue complications. While it is natural to formulate and solve this problem as a multiobjective optimization problem (Küfer et al., 2003), common practice is to determine optimal fluence maps based on the use of a weighted sum of constituent objectives, and proceed in an iterative fashion between the setting of weights and the solution of the resulting optimization problem with a treatment planning software, until a clinically acceptable treatment design has been found. This leads to a time-consuming trial and error process. Therefore, Thieke et al. (2007) proposed a navigation process based on the database approach instead.

Their multiobjective optimization model minimizes a convex combination of the mean dose and the highest doses for each organ at risk as well as two objective functions for the target volume (the tumor). These penalize delivering dose below a prescribed clinical threshold and inhomogeneity of the dose distribution in the target. The search procedure starts by first computing a set of $2^{p-1}$ so-called extreme compromises, which are obtained by minimizing the maximal value of a subset of objectives, for every non-empty subset of the $p$ objectives. This approach is described in more detail in Küfer et al. (2003). The next step in the search procedure is to compute intermediate solutions stochastically, with the goal of collecting a set of points on the Pareto front that are significantly different and equidistant. All these solutions form the database and can be computed without any human involvement.

The determination of a treatment plan is then 
achieved by interactive and online exploration of the database, supported by a graphical user interface that encompasses visualizations of dose distributions and other supporting information. Thieke et al. (2007) used this system for the first time in two clinical test cases (see Figure 4).

We note that another navigation system for radiotherapy treatment design was proposed by Ehrgott and Winz (2007) but never clinically tested.

Railway capacity evaluation: Gandibleux et al. (2010) describe the Multi Criteria Decision Support System RECIFE that supports the evaluation of the capacity of railway infrastructure at the station or junction level. The model's constraints formulation is capable of taking several situations into account: simultaneous stops of several trains on one platform, connecting trains, train coupling or uncoupling, and cyclic timetables for example. This model is structured primarily as a multiobjective set packing problem (Delorme, 2003; Merel, 2012).

The decision process is organized around two major criteria managed lexicographically. Each of them corresponds to a stage of RECIFE. The optimization stage considers the first criterion, which aims to maximize the number of trains scheduled in the timetable. The objective function is optimized with a single objective ant colony optimization algorithm (Gandibleux et al., 2004). A solution is a realistic timetable of $z_{\text {best }}$ trains including different types of trains (e.g., passenger (TGV, IC/IR) and/or freight). Two equivalent solutions have timetables with the same number of trains, but with different types and/or schedules. As output of this optimization stage, the algorithm returns a list $\mathcal{L}$ of the best equivalent solutions found.

The simulation stage follows. It is designed to help the DM to evaluate the stability of the generated timetables and determine the critical items. The second criterion maximizes the stability of equivalent timetables by minimizing the sum of potential delays. Similarly to a domino effect, the principle is based on a propagation model of potential delays (Delorme et al., 2009). Two types of delay are considered, the primary delay caused by a disruption and the secondary delay due to direct or indirect interactions between trains. When a train is delayed, it may produce conflicts with other trains. From the scenario analyzed (kind of traffic, time-windows in the day, density, etc.), the DM, who is usually an expert in railway management, is able to infer reasonable values of primary delay. He evaluates the impact of $p \geq 1$ primary delays, each of them is then viewed as a "dynamically" defined objective, and a corresponding secondary delay is computed for each timetable included in $\mathcal{L}$. All the timetables are evaluated on the $p$ objectives giving their performance. RECIFE allows the user to navigate among the computed Pareto optimal solutions with the support of visual displays such as schedule of trains, space-time graphic, Gantt charts, and animated track views. The output corresponds to one realistic timetable, which maximizes the number of trains using the infrastructure, for the given scenario of traffic, and offering a good stability with regards to possible realistic delays.

Vehicle routing: Applications of the vehicle routing problem (VRP) are typically found in the physical distribution of goods. Customers are visited by vehicles that ship/collect certain goods from/to one or several depots. Obviously, cost criteria are important, with the minimization of the traveled distances as a prominent example of an objective function. Besides, the service provided by the logistical companies comes into play, often being expressed as the agreement of service with promised delivery dates or time windows. Consequently, vehicle routing presents itself as a multiobjective problem, in which the balancing of the considered objectives is of importance (Jozefowiez et al., 2008).

Interactive approaches involving concepts of navigation have recently been adopted to the application domain of the multiobjective VRP. For example, in the work of Geiger and Wenger (2007); Geiger et al. (2007), the DM is given the opportunity to state his/her preferences by means of an overall utility function, combining different objectives into an overall evaluation; Figure 8 shows the graphical user interface allowing the DM to specify his/her preferences. The system then computes a candidate solution maximizing the currently stated utility function, and reports it back to the DM. In a subsequent navigation phase, the DM is allowed to update the utility function prompting the optimization approach to search for 


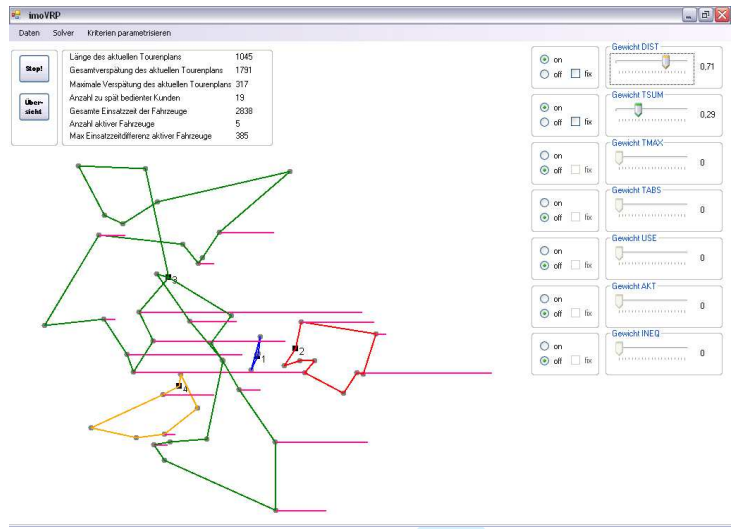

Figure 8: Graphical user interface of a customized navigation method as used in a multiobjective vehicle routing problem.

a new solution. In this spirit, the search for alternatives follows the directions given by the DM. The navigation set at hand depends on the properties of the global utility function. In the case that the utility function employs a convex combination of criteria, the search navigates towards solutions lying on the convex hull of the Pareto front. However, due to the heuristic nature of the implemented optimization approach, which relies on local search, sub-optimal candidate solutions may be presented to the DM too. The interactive search finally terminates when the DM chooses so. In a practical application, this is the case when the DM has visited enough candidate solutions to build his/her preferences, thus converging towards a most-preferred solution.

Flexible manufacturing systems: A flexible manufacturing system (FMS) is a manufacturing system that possesses a certain degree of flexibility to react to changes in the market place and/or customer requirements (Tolio, 2009). The flexibility can relate, for instance, to a system's ability to produce new product types and change the sequence of operations executed (machine flexibility) or the ability to adapt to large-scale changes in, for example, manufacturing volumes and capacities (routing flexibility). However, an FMS is extremely capital-intensive and thus requires careful a priori performance analysis of the system with respect to crucial future managerial, economic and social factors (objectives). A structured decision-making framework can facilitate the FMS design and planning process and, ultimately, optimize these objectives. Pareto race has been used as part of the Visual Interactive Goal Programming (VIG) system (Korhonen, 1987) to assist in the selection process of FMSs (see e.g. Stam and Kuula (1991)). More precisely, after a pre-screening stage at which the set of alternative system configurations is narrowed down to a small number of attractive candidate solutions, Pareto race or another navigation method can be used to explore the remaining configurations in more detail with respect to e.g. capital and running costs, manufacturing flexibility, production volumes and investment risk.

Experimental optimization: An experimental optimization problem is characterized by the feature that the evaluation of candidate solutions involves conducting real experiments, e.g. physical or biochemical experiments, and/or running expensive computer simulations (Box, 1957; Schwefel, 1975; Rechenberg, 2000; Knowles, 2009; Allmendinger and Knowles, 2012; Allmendinger et al., 2015). Examples of such applications include many scientific and technological problems including in areas like drug discovery and manufacturing (Farid, 2007; Small et al., 2011), analytical biochemistry (O'Hagan et al., 2005), experimental quantum control (Shir, 2008), robotics (Harvey et al., 1996), electronics design (Thompson, 1996), food science (Herdy, 1997). The radiotherapy treatment design problem (Ehrgott and Winz, 2007; Thieke et al., 2007) explained in Section 4.2 can also be considered as a type of experimental optimization problem.

In addition to expensive evaluations, experimental optimization problems often involve multiple objectives, limited resources, and user preferences may be available too (further challenges include noisy fitness values, uncertainty, and constraints).

A common situation in experimental optimization is that there is a limited budget and/or fixed project deadline after which it is infeasible or very difficult to perform any additional experiments. Information related to performed experiments is typically stored in 
a database. Consequently, similar to the radiotherapy treatment design problem, navigation can be employed in combination with the database approach to determine a single preferred solutions based on the set of experiments performed upfront.

\section{The Future of Navigation}

In the previous sections we have discussed existing research related to navigation, and also touched upon several real-world applications that have been tackled using navigation. The interesting question now is how can or should navigation evolve to help us even further in future? This section discusses several ideas on how navigation can be made more versatile to address more complex and emerging applications.

Accounting for uncertainty in navigation. Existing navigation methods (see Section 4.1) assume (or ignore) that the optimization problem at hand is deterministic. Unfortunately, this condition is rarely the case, especially when dealing with real-world optimization problems. Of course, if we knew the probability distributions governing the uncertainty, then we could replace the underlying optimization procedure with one that is capable of accounting for uncertainty, such as a stochastic linear programming (Kall and Mayer, 2010). If the number of potential (uncertain) scenarios is very large or the probability distributions are unknown, then the optimization procedure can be linked with Monte Carlo (MC) sampling. This approach becomes trickier when dealing with expensive optimization problems as MC trials can become costly and/or time-consuming. Also, depending on the navigation method, e.g. the database approach, we may need to perform MC trials upfront. This leads to the next question, namely how to represent uncertainty in an intuitive way for the DM so as to affect the navigation direction and/or speed.

Accounting for many objectives in navigation. Current applications of navigation methods are limited to problems with up to around five objectives. However, of course, problems can have many more objectives (Pilat and Neruda, 2013; Fleming et al., 2005); in the evolutionary multiobjective community, such problems are referred to as many-objective optimization problems (Ishibuchi et al., 2008). Navigating in a space with many objectives is challenging from both an optimization algorithm and user perspective as information from a potentially large number of trade-offs needs to be processed to guide the navigation process meaningfully. To support the user in the navigation process of such problems, the trade-offs between objectives need to be presented in a more compact and intuitive way. For example, it may be possible to group objectives according to their importance in order to navigate through a lower-dimensional space. Another option could be to employ a hierarchical approach where navigation is applied sequentially to different sets of objectives until all objectives have been processed. Finally, if we think of navigation as a sort of clustering approach, then the application of (multiobjective) clustering methods (Handl and Knowles, 2007; Branke et al., 2008) may also prove promising to cope with many objectives. In any case, adjusting the navigation method and user interface to the underlying objective optimization algorithm seems more crucial in the case of many objectives.

Facilitating navigation through the design of more intuitive graphical user interfaces (GUIs). To widen both the application area and user group of navigation methods, it is vital to establish GUIs that allow not only for an intuitive interaction with the system but also visualize relevant search-related information to a DM. More efficient user interfaces become more crucial as the complexity of problems increases, e.g. because of uncertainty and many objectives. To develop more intuitive and informative user interfaces we can get inspiration and borrow ideas from related research fields, such as visual analytics (Wong and Thomas, 2004), game design (Johnson and Wiles, 2003), human computer interaction (Shneiderman, 1992). Furthermore, to ensure that the development of the GUI is on the right track, the algorithm designer may want to involve both visualization experts and end users of the final navigation method. The MCDM community has started to look at some of these ideas recently (Hakanen and Miettinen, 2015).

Emerging application domains of navigation. Cur- 
rent research has developed navigation methods primarily for problems where the objective functions are known in closed form allowing the exploitation of gradient information (which is in fact typically done). However, there are also problems, such as experimental optimization problems, where this information is not available because the objective function values have to be determined via a physical experiment or a time-consuming computer simulation. While a database approach is feasible to cope with such problems (see above), this approach is static in the sense that the navigation set is fixed during the search procedure. One way to make navigation more interactive for such problems, while keeping the number of expensive evaluations at a minimum, is to use a surrogateassisted multiobjective optimization algorithm (Jin, 2011) to determine the solution(s) presented to the DM.

\section{Summary}

Selecting the single most-preferred solution from a set of candidate solutions is an intrinsically complex task not only because there is limited understanding about the problem at hand but also due to the presence of incomplete, approximate, uncertain or fuzzy user preferences. An interactive procedure that traverses through a set of points can aid in the process of finding a single most-preferred solution.

In our previous preliminary work (Allmendinger et al., 2012), we have introduced the umbrella term navigation to refer to such interactive procedures. This work extends our work by providing a more formal and comprehensive overview on navigation and related research. More precisely, we have described a general framework to capture a wide range of navigation methods, described the key aspects of navigation and discussed several features according to which navigation methods can be characterized. Following this, we have put the framework in a proper context by linking it with existing navigation methods and real-world problems to which these methods have been applied. Finally, we have highlighted directions of future research including (i) widening the application of navigation methods to more complex and emerging problem do- mains, such as problems with uncertainty and many objectives, (ii) development of more intuitive and informative graphical user interfaces, and (iii) the development of more efficient navigation methods based on emerging techniques, such as surrogate-based methods.

\section{Acknowledgement}

This paper is a product of discussions initiated in the Dagstuhl Seminar 12041: Learning in Multiobjective Optimization. The authors acknowledge gratefully Prof. Kaisa Miettinen and Dr. Jussi Hakanen for contributing to these discussions.

\section{References}

Allmendinger, R., Braun, H., Ehrgott, M., Gandibleux, X., Geiger, M. J., Klamroth, K., Korhonen, P., Luque, M., and Zitzler, E. (2012). Navigation in multi objective optimization methods. In Greco, S., Knowles, J. D., Miettinen, K., and Zitzler, E., editors, Learning in Multiobjective Optimization (Dagstuhl Seminar 12041), pages 86-92, LeibnizZentrum für Informatik Schloss Dagstuhl, Germany.

Allmendinger, R., Handl, J., and Knowles, J. (2015). Multiobjective optimization: When objectives exhibit unequal latencies. European Journal of Operational Research, 243(2):497-513.

Allmendinger, R. and Knowles, J. (2012). On handling ephemeral resource constraints in evolutionary search. Evolutionary Computation, 21(3):497531.

Box, G. E. P. (1957). Evolutionary operation: A method for increasing industrial productivity. $A p$ plied Statistics, 6(2):81-101.

Branke, J., Corrente, S., Greco, S., Słowiński, R., and Zielniewicz, P. (2016). Using Choquet integral as preference model in interactive evolutionary multiobjective optimization. European Journal of Operational Research, 250(3):884-901. 
Branke, J., Deb, K., Miettinen, K., and Słowiński, R., editors (2008). Multiobjective Optimization: Interactive and Evolutionary Approaches, volume 5252 of Lecture Notes in Computer Science. Springer, Berlin Heidelberg.

Branke, J., Greco, S., Słowiński, R., and Zielniewicz, P. (2010). Interactive evolutionary multiobjective optimization driven by robust ordinal regression. Bulletin of the Polish Academy of Sciences: Technical Sciences, 58(3):347-358.

Branke, J., Greco, S., Słowiński, R., and Zielniewicz, P. (2015). Learning value functions in interactive evolutionary multiobjective optimization. IEEE Transactions on Evolutionary Computation, 19(1):88-102.

Chen, L. and Pu, P. (2004). Survey of preference elicitation methods. Technical report, Swiss Federal Institute of Technology in Lausanne, Technical Report No. IC/200467.

Deb, K. (2001). Multi-objective optimization using evolutionary algorithms, volume 16 of WileyInterscience Series in Systems and Optimization. John Wiley \& Sons, Chichester.

Delorme, X. (2003). Modélisation et résolution de problèmes liés à l'exploitation d'infrastructures ferroviaires. $\mathrm{PhD}$ thesis, Université de Valenciennes et du Hainaut Cambrésis, France.

Delorme, X., Gandibleux, X., and Rodriguez, J. (2009). Stability evaluation of a railway timetable at station level. European Journal of Operational Research, 195(3):780-790.

Ehrgott, M., Güler, Ç., Hamacher, H. W., and Shao, L. (2009). Mathematical optimization in intensity modulated radiation therapy. Annals of Operations Research, 175(1):309-365.

Ehrgott, M. and Winz, I. (2007). Interactive decision support in radiation therapy treatment planning. $O R$ Spectrum, 30(2):311-329.
Eskelinen, P., Miettinen, K., Klamroth, K., and Hakanen, J. (2010). Pareto navigator for interactive nonlinear multiobjective optimization. OR Spectrum, 23(1):211-227.

Farid, S. S. (2007). Process economics of industrial monoclonal antibody manufacture. Journal of Chromatography B, 848(1):8-18.

Faulkenberg, S. L. and Wiecek, M. M. (2010). On the quality of discrete representations in multiple objective programming. Optimization and Engineering, 11(3):423-440.

Faulkenberg, S. L. and Wiecek, M. M. (2012). Generating equidistant representations in biobjective programming. Computational Optimization and Applications, 51(3):1173-1210.

Fleming, P. J., Purshouse, R. C., and Lygoe, R. J. (2005). Many-objective optimization: An engineering design perspective. In Coello Coello, C. A., Hernández Aguirre, A., and Zitzler, E., editors, Evolutionary Multi-Criterion Optimization, volume 3410 of Lecture Notes in Computer Science, pages 14-32. Springer Berlin Heidelberg.

Gandibleux, X., Delorme, X., and T'Kindt, V. (2004). An ant colony algorithm for the set packing problem. In Dorigo, M., Birattari, M., Blum, C., Gambardella, L., Mondada, F., and Stützle, T., editors, Ant Colony Optimization and Swarm Intelligence, volume 3172 of Lecture Notes in Computer Sciences, pages 49-60. Springer, Berlin Heidelberg.

Gandibleux, X., Riteau, P., and Delorme, X. (2010). RECIFE: A MCDSS for railway capacity evaluation. In Ehrgott, M., Naujoks, B., Stewart, T., and Wallenius, J., editors, Multiple Criteria Decision Making for Sustainable Energy and Transportation Systems, volume 634 of Lecture Notes in Economics and Mathematical Systems, pages 93-103. Springer, Berlin Heidelberg.

Geiger, M. J. and Wenger, W. (2007). On the interactive resolution of multi-objective vehicle routing problems. In Obayashi, S., Deb, K., Poloni, 
C., Hiroyasu, T., and Murata, T., editors, Evolutionary Multi-Criterion Optimization: 4th International Conference, EMO 2007, volume 4403 of Lecture Notes in Computer Science, pages 687-699. Springer, Berlin Heidelberg.

Geiger, M. J., Wenger, W., and Habenicht, W. (2007). Interactive utility maximization in multi-objective vehicle routing problems: A "decision maker in the loop"-approach. In Proceedings of the 2007 IEEE Symposium on Computational Intelligence in Multicriteria Decision Making (MCDM 2007), pages 178-184. IEEE.

Gnanadesikan, R. (2011). Methods for Statistical Data Analysis of Multivariate Observations, volume 321 of Wiley Series in Probability and Statistics. John Wiley \& Sons, Chichester.

Greco, S., Ehrgott, M., and Figueira, J., editors (2016). Multiple Criteria Decision Analysis: State of the Art Surveys. International Series in Operations Research \& Management Science. Springer, New York, second edition.

Greenwood, G., Hu, X., and D'Ambrosio, J. (1997). Fitness functions for multiple objective optimization problems: Combining preferences with Pareto rankings. In Belew, R. and M.D., V., editors, Foundations of Genetic Algorithms, pages 437-455. Morgan Kaufmann, San Mateo.

Haimes, Y., Lasdon, L., and Wismer, D. (1971). On a bicriterion formulation of the problems of integrated system identification and system optimization. IEEE Transactions on Systems Man and Cybernetics, 1:296-297.

Hakanen, J. and Miettinen, K. (2015). Using interaction techniques in implementing interactive multiobjective optimization methods. In 23rd International Conference on Multiple Criteria Decision Making - Book of Abstracts, page 112.

Handl, J. and Knowles, J. (2007). An evolutionary approach to multiobjective clustering. Evolutionary Computation, IEEE Transactions on, 11(1):56-76.
Hartikainen, M., Miettinen, K., and Klamroth, K. (2015). An interactive method for multiobjective optimization: Nonconvex Pareto navigator. Technical Report Series B, Scientific Computing, No. B 3/2015, Department of Mathematical Information Technology, University of Jyväskylä, Finland.

Hartikainen, M., Miettinen, K., and Wiecek, M. M. (2012). Pareto front interpolation for nonlinear multiobjective optimization. Computational Optimization and Applications, 52(3):845-867.

Harvey, I., Husbands, P., Cliff, D., Thompson, A., and Jakobi, N. (1996). Evolutionary robotics: The Sussex approach. Robotics and Autonomous Systems, 20(2-4):205-224.

Herdy, M. (1997). Evolutionary optimization based on subjective selection - Evolving blends of coffee. In EUFIT'97 5th European Congress on Intelligent Techniques and Soft Computing, pages 640-644.

Ishibuchi, H., Tsukamoto, N., and Nojima, Y. (2008). Evolutionary many-objective optimization: A short review. In IEEE Congress on Evolutionary Computation, pages 2419-2426. IEEE.

Jin, Y. (2011). Surrogate-assisted evolutionary computation: Recent advances and future challenges. Swarm and Evolutionary Computation, 1(2):61-70.

Johnson, D. and Wiles, J. (2003). Effective affective user interface design in games. Ergonomics, 46(1314):1332-1345.

Jozefowiez, N., Semet, F., and Talbi, E.-G. (2008). Multi-objective vehicle routing problems. European Journal of Operational Research, 189(2):293-309.

Kahneman, D. and Tversky, A. (1979). Prospect theory: An analysis of decision under risk. Econometrica: Journal of the Econometric Society, 47(2):263-291.

Kall, P. and Mayer, J. (2010). Stochastic Linear Programming - Models, Theory, and Computation. Springer, Berlin Heidelberg, second edition. 
Keeney, R. L. and Raiffa, H. (1976). Decisions with Multiple Objectives: Preferences and Value Tradeoffs. Cambridge University Press, Cambridge.

Knowles, J. (2009). Closed-loop evolutionary multiobjective optimization. IEEE Computational Intelligence Magazine, 4(3):77-91.

Korhonen, P. (1987). VIG - A visual interactive support system for multiple criteria decision making. Belgian Journal of Operations Research, Statistics and Computer Science, 27:3-15.

Korhonen, P. and Laakso, J. (1986a). Solving generalized goal programming problems using a visual interactive approach. European Journal of Operational Research, 26(3):355-363.

Korhonen, P. and Wallenius, J. (1988). A Pareto race. Naval Research Logistics, 35(6):615-623.

Korhonen, P. J. and Laakso, J. (1986b). A visual interactive method for solving the multiple criteria problem. European Journal of Operational Research, 24(2):277-287.

Korhonen, P. J. and Yu, G. Y. (1997). Quadratic Pareto race. Technical Report IR-97-58, International Institute for Applied Systems Analysis, Laxenburg, Austria.

Küfer, K., Scherrer, A., Monz, M., Alonso, F., Trinkaus, H., Bortfeld, T., and Thieke, C. (2003). Intensity-modulated radiotherapy - A large scale multi-criteria programming problem. OR Spectrum, 25(2):223-249.

Lotov, A. V., Kamenev, G. K., Berezkin, V. E., and Miettinen, K. (2005). Optimal control of cooling process in continuous casting of steel using a visualization-based multi-criteria approach. Applied Mathematical Modelling, 29(7):653-672.

Lotov, A. V. and Miettinen, K. (2008). Visualizing the Pareto frontier. In Multiobjective optimization, pages 213-243. Springer, Berlin Heidelberg.

Malakooti, B. and Raman, V. (2000). Clustering and selection of multiple criteria alternatives using unsupervised and supervised neural networks. Journal of Intelligent Manufacturing, 11(5):435-451.
Merel, X. (2012). Evaluation biobjectif de la capacité d'infrastructures ferroviaires par génération de colonnes hybride. $\mathrm{PhD}$ thesis, Université de Nantes, France.

Miettinen, K. (1999). Nonlinear Multiobjective Optimization. Kluwer Academic Publishers, Boston London Dordrecht.

Miettinen, K. (2014). Survey of methods to visualize alternatives in multiple criteria decision making problems. OR Spectrum, 36(1):3-37.

Miettinen, K., Eskelinen, P., Ruiz, F., and Luque, M. (2010). NAUTILUS method: An interactive technique in multiobjective optimization based on the nadir point. European Journal of Operational Research, 206(2):426-434.

Miettinen, K. and Mäkelä, M. M. (1995). Interactive bundle-nased method for nondifferentiable multiobjective optimization: NIMBUS. Optimization, 34(3):231-246.

Miller, G. (1956). The magical number seven, plus or minus two: some limits on our capacity for processing information. Psychological Review, 63(2):81.

O’Hagan, S., Dunn, W. B., Brown, M., Knowles, J., and Kell, D. B. (2005). Closed-loop, multiobjective optimization of analytical instrumentation: Gas chromatography / time-of-flight mass spectrometry of the metabolomes of human serum and of yeast fermentations. Analytical Chemistry, 77(1):290 303.

Pilat, M. and Neruda, R. (2013). Aggregate metamodels for evolutionary multiobjective and manyobjective optimization. Neurocomputing, 116:392402.

Purshouse, R., Deb, K., Mansor, M., Mostaghim, S., and Wang, R. (2014). A review of hybrid evolutionary multiple criteria decision making methods. In IEEE Congress on Evolutionary Computation, pages 1147-1154. IEEE.

Rechenberg, I. (2000). Case studies in evolutionary experimentation and computation. Computer 
Methods in Applied Mechanics and Engineering, 186(2):125-140.

Ruiz, A., Sindhya, K., Miettinen, K., Ruiz, F., and Luque, M. (2015a). E-NAUTILUS: A decision support system for complex multiobjective optimization problems based on the nautilus method. European Journal of Operational Research, 246(1):218231.

Ruiz, A. B., Luque, M., Miettinen, K., and Saborido, R. (2015b). An interactive evolutionary multiobjective optimization method: Interactive WASF-GA. In Gaspar-Cunha, A., Henggeler Antunes, C., and Coello Coello, C. A., editors, Evolutionary MultiCriterion Optimization Part II, volume 9019 of Lecture Notes in Computer Science, pages 249-263. Springer, Berlin Heidelberg.

Saaty, T. L. (1977). A scaling method for priorities in hierarchical structures. Journal of Mathematical Psychology, 15(3):234-281.

Sayın, S. (2000). Measuring the quality of discrete representations of efficient sets in multiple objective mathematical programming. Mathematical Programming, 87(3):543-560.

Schwefel, H.-P. (1975). Evolutionsstrategie und $\mathrm{Nu}$ merische Optimierung. $\mathrm{PhD}$ thesis, Technical University of Berlin, Germany.

Shir, O. M. (2008). Niching in Derandomized Evolution Strategies and Its Applications in Quantum Control: A Journey from Organic Diversity to Conceptual Quantum Designs. PhD thesis, University of Leiden, The Netherlands.

Shneiderman, B. (1992). Designing the user interface: strategies for effective human-computer interaction. Addison-Wesley, Reading.

Small, B. G., McColl, B. W., Allmendinger, R., Pahle, J., López-Castejón, G., Rothwell, N. J., Knowles, J., Mendes, P., Brough, D., and Kell, D. B. (2011). Efficient discovery of anti-inflammatory small molecule combinations using evolutionary computing. Nature Chemical Biology, 7(12):902-908.
Stam, A. and Kuula, M. (1991). Selecting a flexible manufacturing system using multiple criteria analysis. International Journal of Production Research, 29(4):803-820.

Thieke, C., Küfer, K., Monz, M., Scherrer, A., Alonso, F., Oelfke, U., Huber, P., Debus, J., and Bortfeld, T. (2007). A new concept for interactive radiotherapy planning with multicriteria optimization: First clinical evaluation. Radiotherapy and Oncology, 85(2):292-298.

Thompson, A. (1996). Hardware Evolution: Automatic design of electronic circuits in reconfigurable hardware by artificial evolution. PhD thesis, University of Sussex, United Kingdom.

Tolio, T., editor (2009). Design of Flexible Production Systems: MethodoMethod and Tools. Springer, Berlin Heidelberg.

Triantaphyllou, E. (2000). Multi-criteria Decision Making Methods: A Comparative Study, volume 44 of Applied Optimization. Springer, Berlin Heidelberg.

Wierzbicki, A. P. (1980). The use of reference objectives in multiobjective optimization. In Fandel, G. and Gal, T., editors, Multiple Criteria Decision Making Theory and Application, volume 177 of Lecture Notes in Ecinomics and Mathematic Systems, pages 468-486. Springer, Berlin Heidelberg.

Wong, P. C. and Thomas, J. (2004). Visual analytics. IEEE Computer Graphics and Applications, 24(5):20-21. 